\title{
Detection of cavitation events upon freezing and thawing of water in stems using ultrasound techniques
}

\author{
A. Raschi', G.E. Scarascia- Mugnozza², R. Valentini2 \\ and C. Vazzana ${ }^{3}$ \\ 1 C.N.R.-I.A.T.A., 50144 Firenze, \\ 2 Ist. Biol. Selvicolturale - Univ. della Tuscia, Viterbo, and \\ ${ }^{3}$ Dipt. di Agronomia e Produzioni Erbacee, Univ. di Firenze, P.le delle Cascine, 18, Firenze, Italia
}

\section{Introduction}

In recent years, renewed attention has been paid to cavitative events occurring in plants subjected to water stress. This has been a result of the creation of new techniques based on the detection of sounds (Milburn, 1973) or, more reliably, ultrasounds (Tyree and Dixon, 1983) emitted by the plant as a consequence of the vibration of cavitating conducting elements. The results obtained so far show that the development of emboli is a phenomenon that occurs much more frequently than was supposed, and takes place at a different frequency in different species. Indeed, several observations, summarized by Zimmermann (1983), suggest that cavitation should take place upon the freezing and the thawing of water in vessels. In fact, the existence of cavitative events as a consequence of cycles of freezing and thawing has been evidenced by several authors (Scholander et al., 1955; Hammel, 1967; Sucoff, 1969), who showed the existence of permanent damage to the conductive system in some species. To date, research has investi- gated the consequences of the above mentioned cycles, without directly demonstrating the cavitative events as they were taking place. Moreover, most of the work has been carried out on conifers, which, according to the literature, are less damaged than angiosperms. In this preliminary text, we examine the differences in the ultrasound emissions that exist between a coniferous (Araucaria excelsa) and a broad-leafed (Eucalyptus occidentalis) tree during lowering and rising of the temperature.

\section{Materials and Methods}

The experiment was carried out in the laboratory of the Agronomy Department of the University of Firenze, Italy, using 3 yr old potted plants of E. occidentalis Endl., and $4 \mathrm{yr}$ old excised lateral branches of $A$. excelsa $\mathrm{R}$. Br. All plants had been acclimated for 2 wk in a growth chamber and none of them had experienced water stress or freezing during the previous months; xylem water potential, measured by pressure chamber on all plants before the experiments, ranged between 0.07 and $0.11 \mathrm{MPa}$. The pots of Eucalyptus plants were enclosed in a thermal 
insulated box to prevent soil freezing. The cut extremity of Auracaria branches, recut under water, was kept immersed in water and enclosed in an insulated box. Plant stems were frozen in a top loading freezer, whose top cover had been substituted with expanded polystyrene material. A hole had been cut in the new cover in order to allow the distal part of the sample, with all leaves to be kept at room temperature, enclosed in a black plastic bag. Temperature was lowered at a rate of $5^{\circ} \mathrm{C}$ per hour. To measure the acoustic emission rate, a Bruel and Kjaer 8312 broad band sensor was clamped in a handpiece to the lower part of the stem, which had previously been prepared by removing a small window of bark to expose the xylem. This area was coated with petroleum jelly to prevent water loss and then with ultrasound gel to improve acoustic transmission. To avoid heating effects of the transducer on the sensor and the sample, we used the sensor intermittently, switching it on only for 2 min out of 10 . The acoustic emissions were counted using an instrument similar to the one described by Sandford and Grace (1985), slightly modified. Freezing of stem tissues was monitored with thermocouple sensors (type T) connected to a strip chart recorder.

\section{Results}

In Fig. 1, the typical behavior of ultrasound pulses as a function of stem temperature in Eucalyptus is reported. Before ice formation, which occurred at an average supercooling temperature of $-4.5^{\circ} \mathrm{C}$, only a few isolated events were recorded. A considerable emission rate appeared several minutes after ice formation and the maximum was detected at the lowest temperature reached $\left(-8^{\circ} \mathrm{C}\right)$. As the temperature was increased, ultrasound emission gradually diminished, while upon thawing we detected only a few counts.

Fig. 2 presents the behavior of ultrasound emissions in Araucaria subjected to the same kind of test. As in Eucalyptus, only a few single events were recorded during supercooling, but the highest number of acoustic events were detected a few minutes after ice formation or imme-
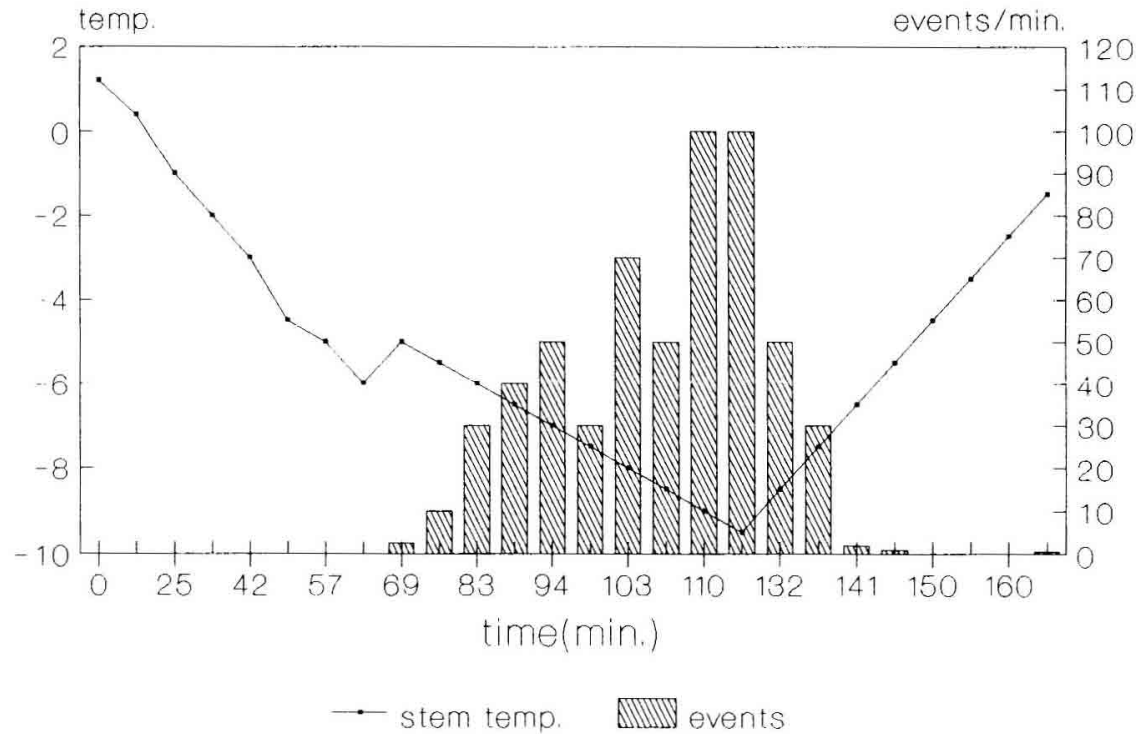

Fig. 1. Typical behavior of acoustic emissions in Eucalyptus as a function of stem temperature. 


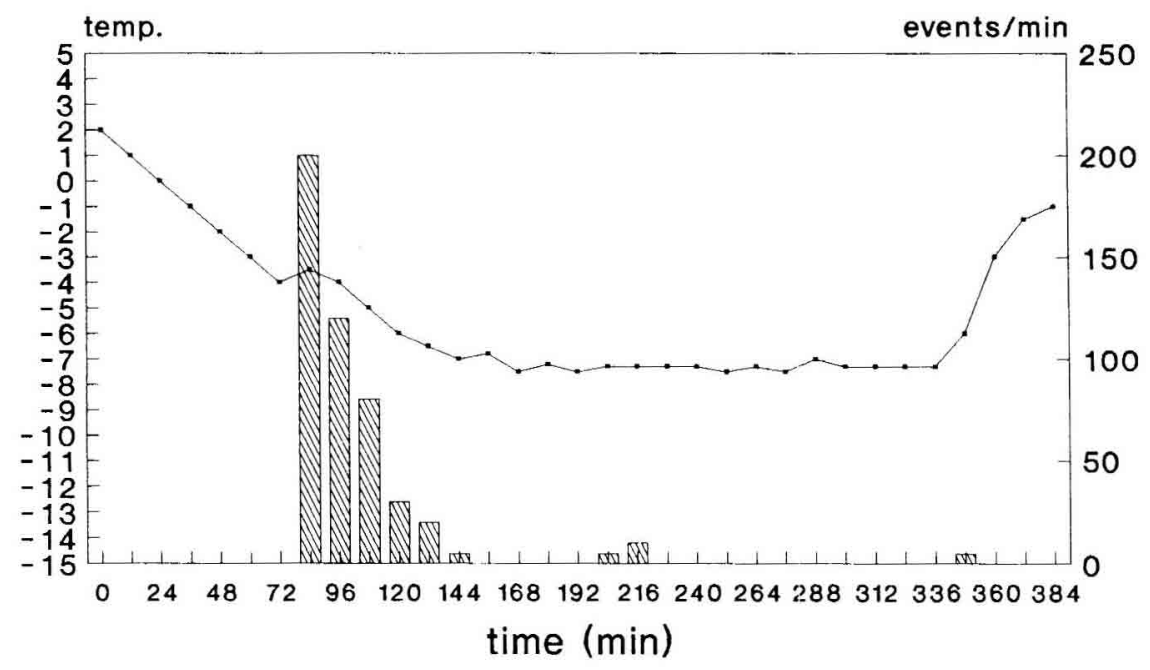

- Stem temp \$MUW Events

Fig. 2. Typical behavior of acoustic emissions in Araucaria as a function of stem temperature.

diately atter it. Emission rates then decreased, coming to zero in about $1 \mathrm{~h}$. During thawing, the trend was similar to that observed in Eucalyptus plants.

\section{Discussion and Conclusions}

In water-stressed plants, it is generally agreed that a single emission represents the cavitation of water in one vessel. Doubts exist about the counting efficiency of this kind of sensor, which is thought to be quite low. In our tests, no events were recorded using the sensor alone in the freezer (i.e., the environmental noise was low) or freezing a dehydrated piece of stem. Moreover, the detected events appeared as groups, divided by periods of silence with just a few isolated events, as they appear during water stress. Therefore, we concluded that the recorded events are really due to cavitation. Previous research has showed the different behavior of angiosperms, which develop emboli as a consequence of these cycles, and conifers in which xylem functionality is not lost. Reasons for the different behavior patterns should be found in wood structure, since the bordered pits on the tracheids can isolate the freezing sap, preventing the expansion of emboli to the adjacent vessels. The behavior of acoustic emissions in Eucalyptus could be linked to the fact that freezing does not take place at the same time in all the elements of wood but is somewhat progressive. It can also be explained by a dehydration process caused by the presence of extracellular ice, which is responsible for an external force withdrawing water from cells. Since we were working at saturated atmospheric water potential, we are unable to make reliable conjectures about the behavior of cavitation in a frozen stem under field conditions. 
Further research is needed to assess that, together with examining the modifications in water uptake and water conductivity during freezing and after thawing.

\section{References}

Hammel H.T. (1967) Freezing of xylem sap without cavitation. Plant Physiol. 42, 55-66

Milburn J.A. (1973) Cavitation studies on whole ricinus plants by acoustic detection. Planta 112, 333-342
Sandford A.P. \& Grace J. (1985) The measurement and interpretation of ultrasound from woody stems. J. Exp. Bot. 36, 298-311

Scholander P.F., Love W.E. \& Kanwsher J.W. (1955) The rise of sap in tall grapevines. Plant Physiol. 30, 93-104

Sucoft E. (1969) Freezing of conifer xylem and the cohesion tension theory. Physiol. Plant. 22, 424-431

Tyree M.T. \& Dixon M.A. (1983) Cavitation events in Thuja occidentalis? Ultrasonic acoustic emission from the sapwood can be measured. Plant Physiol. 72, 1094-1099

Zimmermann M.H. (1983) In: Xylem Structure and the Ascent of Sap. Springer-Verlag, Berlin, pp. 143 